\title{
3-D Low-Loss Coplanar Waveguide Transmission Lines in Multilayer MMICs
}

\author{
Van Tuyen Vo, Lokesh Krishnamurthy, Student Member, IEEE, Qing Sun, Student Member, IEEE, and \\ Ali A. Rezazadeh, Member, IEEE
}

\begin{abstract}
Newly developed transmission-line structures using the great flexibility of three-dimensional multilayer technology have been designed and fabricated. In this paper, we demonstrate that monolithic microwave integrated circuit (MMIC) coplanar waveguide transmission lines with a wide range of characteristic impedances can easily be designed using the multilayer technique. Furthermore, this implementation can avoid the well-known current crowding effects on the conductor edges minimizing dissipation loss. The system of three layers of metals and two layers of sandwich polyimide as dielectrics was employed. The fabricated transmission lines have been characterized providing a wide range of impedances from 10 to $70 \Omega$. In addition, the effects of unintentional horizontal and vertical coupling in multilayer MMICs have been investigated. The results indicated that an optimum separation of $75 \mu \mathrm{m}$ is necessary for negligible coupling $(\sim-30 \mathrm{~dB})$.
\end{abstract}

Index Terms-Coplanar waveguide (CPW), coupling, monolithic microwave integrated circuits (MMICs), transmission lines.

\section{INTRODUCTION}

$\mathbf{T}$ HERE HAS been an increasing demand in microwave integrated-circuit technologies to lower the dissipation loss of transmission lines in order to improve the noise figure and efficiency performance of such circuits like low-noise and power amplifiers. Low-loss transmission lines are also desirable to create low-loss passive components such as filters, baluns, power divider/combiners, and couplers. The coplanar waveguide (CPW) is an alternate to microstrip concept [1], [2], [7], [8]. In CPW design, via-holes are not necessary and fragile semiconductors do not need to be excessively thin. In addition, the individual component sizes are unlimited. These factors can greatly reduce the processing costs. CPW concept is well suited for use with field-effect transistors such as pseudomorphic high electron-mobility transistors (pHEMTs), where RF grounding must be close to the device [1]. CPW transmission lines are also less lossy than microstrip ones [2], [7]. For several applications, such as reduced-size couplers and nonlinear transmission lines, it is desirable to use high-impedance lines [3]. To increase the characteristic impedance of CPWs, the gap must be widened and the center conductor reduced, which has the effect of increasing the conductivity loss. Elevation of the

Manuscript received October 10, 2005; revised February 20, 2006. This work was supported by the Electro-Magnetic Remote Sensing Defence Technology Centre, established by the U.K. Ministry of Defence and run by a consortium of BAE Systems Avionics, Thales Defence, Roke Manor Research, and Filtronic.

The authors are with the School of Electrical and Electronic Engineering, University of Manchester, Manchester M60 1QD, U.K. (e-mail: tuyen.vo@ manchester.ac.uk)

Digital Object Identifier 10.1109/TMTT.2006.875458

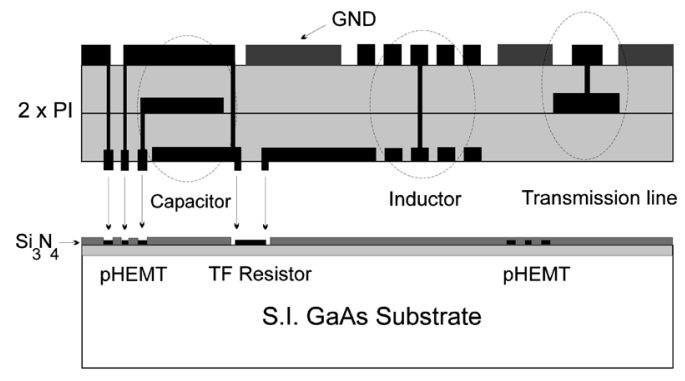

Fig. 1. Cross-sectional view of a 3-D MMIC incorporating passive CPW multilayer and GaAs pHEMT technologies.

center conductor, however, can increase the impedance without reducing the center conductor width [9], [10], [13]. On the other hand, ultra-low impedance transmission lines are needed in matching networks where low-impedance devices such as power field-effect transistors (FETs) or photodiodes are used. However, the range of the characteristic impedance of conventional CPWs is limited. The maximum impedance is limited by the practical size of the slot and the width of the center line, while a practical low limit is imposed by fabrication of the very narrow slot, and the high losses resulting from current crowding at the conductors edges. The conductor gapwidth limitation and high current crowding are overcome by employing a V-shaped center conductor [12]. This structure can eliminate the current concentration at the edge of the conductor. The center conductor can also be extended under the ground planes, resulting in higher capacitance per unit length and, thus, lowering the characteristic impedance.

One problem associated with the $\mathrm{CPW}$ is that the ground must be on either side of the signal lines, which increases the complexity of the circuit designs. A potential solution to this is to utilize a multilayer technique in which several metal layers are sandwiched by insulators. This approach gives microwave engineers the flexibility in designing multilayer structures with improved circuit performance.

The recent interest in highly integrated monolithic microwave integrated circuit (MMIC) for wireless application has been driven by the expansion of the market for wireless communications and sensors. Recently, the thin-film multilayer technology demonstrates that it can be very effective in realization of miniaturization and high-level integration, which results in reduction of chip size and, thus, low cost [11], [13]. A threedimensional (3-D) MMIC, shown in Fig. 1, is a promising technology for flexible design and cost-effective fabrication of millimeter-wave MMICs. In this structure, active devices such as pHEMT resistors are formed on a semi-insulating 
GaAs substrate, which carries multilayer of conductors and sandwich dielectrics. CPW transmission-line interconnects, passive components such as capacitors, inductors, coupler, baluns, and matching circuits are built into these multilayers. Such technology reduces the size of devices like inductors by approximately $75 \%$, and significantly improves electrical performance while reducing cost. This technology also offers an effective separation of the application circuit process from the semiconductor active device process, resulting in a much shorter turnaround time.

However, in order to keep the MMIC chip area small, the separation between adjoining CPW transmission lines should be as small as possible, which may create parasitic coupling between the transmission lines. It can also be a problem if there is unintentional vertical coupling between CPW transmission lines and conductor elements of different metal layers [14]-[16].

In this paper, we present a complete set of low-loss 3-D CPW transmission lines with characteristic impedance ranging from as low as $10-50$ to $70 \Omega$ [17], which are ready for integration with other passive and active components. The impedance can be extended up to $100 \Omega$ easily by changing dimension of transmission lines. Fig. 2 shows the cross-sectional view of various structures of CPW lines using the great flexibility of 3-D multilayer technology. These structures do not require special fabrication techniques and any combination of them can be created on any substrate and also incorporated with any active device technology. In this study, several multilayer CPW transmission lines have been fabricated and characterized on semi-insulating GaAs substrates. The results show that a significant improvement can be achieved using multilayer structures compared with the conventional microstrip MMIC concept.

Furthermore, the discussion is extended to include the effects of unintentional coupling both vertically and horizontally in multilayer MMICs. An evaluation of the unintentional coupling between CPW transmission lines and other conductor elements including metal contacts of active devices that are embedded in polyimide layers or built on semi-insulating GaAs is presented for the first time. Simulations and experimental measurements are used for this evaluation. The results suggest an optimum separation between adjoining CPW transmission lines where electromagnetic coupling can be neglected, i.e., lower than $-30 \mathrm{~dB}$. Simulation of the vertical coupling effect on characteristics of CPW transmission was also carried out and the results have been discussed.

\section{3-D CPW TRANSMISSION LINES}

The multilayer CPW transmission lines in this study have been fabricated using three layers of metals and two layers of sandwich dielectrics. In realizing these multilayer structures, several processing aspects have been studied including polyimide spin, curing, etching, and metal contact formation. In these structures, different layers need to be interconnected properly through the etched windows of the polyimide insulating layers. The thickness of Au layers (M1, M2, and M3) was approximately $0.8 \mu \mathrm{m}$. The isolating polyimide layers between metal layers was $2.5-\mu \mathrm{m}$ thick, and the semi-insulating GaAs substrate was approximately $600 \mu \mathrm{m}$. The polyimide used in this study has a dielectric constant of approximately 3.7. A conventional planar CPW transmission line with the center conductor width $W=20 \mu \mathrm{m}$ and slot width $G=15 \mu \mathrm{m}$ was chosen as a basic structure, which is a compromise between the loss and compactness. The top center conductor of low-impedance transmission lines was made wider $(W=40 \mu \mathrm{m})$ in order to reduce the impedance and also compensate increased dielectric loss due to the increased electric field in the overlapping area where the distance between the center line and ground becomes very close, only a few micrometers. All the transmission lines are 2-mm long.

The polyimide interconnection windows were formed by oxygen plasma reactive ion etching (RIE) through a photoresist protecting layer patterned using the lithography process. In order to optimize the polyimide etching process, different polyimide etching conditions have been tried including varying plasma power, chamber pressure, and gas flow rate.

The fabricated multilayer CPW transmission lines were characterized at microwave frequencies using a Cascade Microtech on-wafer probe station and an HP 8510B vector network analyzer.

The frequency dependence of transmission-line parameters such as characteristic impedance, effective dielectric constant, and dissipation loss are calculated from the measured $S$-parameters [4]-[8]. Based on the solution of the classical Telegrapher's transmission-line equation, the characteristic impedance $Z_{0}$ can be expressed as [4]

$$
Z_{0}=Z_{\text {sys }} \sqrt{\frac{\left(1+S_{11}\right)^{2}-S_{21}^{2}}{\left(1-S_{11}\right)^{2}-S_{21}^{2}}}
$$

where $Z_{\text {sys }}=50 \Omega$ is the system impedance.

The effective dielectric constant can be calculated from the imaginary part $(\beta)$ of the propagation constant [4]

$$
\varepsilon_{r, \text { effective }}=\left(\frac{\beta c}{2 \pi f}\right)^{2}
$$

where $c$ is the speed of light in space.

It is useful to plot the variation of dissipation loss factor with frequency for these multilayer structures. It is very common to consider the use of a two-port network where the transmitted power to the device is $1-\left|S_{11}\right|^{2}$. The output power can be approximated as $\left|S_{21}\right|^{2}$. Therefore, the dissipation loss can be approximated by

$$
\text { Loss }=10 \log \left(\frac{1-\left|S_{11}\right|^{2}}{\left|S_{21}\right|^{2}}\right) .
$$

The geometry and dimension of transmission lines in this study were analyzed and optimized with the help of the two-and-one-half-dimensional (2.5-D) electromagnetic simulator Momentum, which is a part of the ADS 2004A software.

\section{A. 50- $\Omega$ Transmission Lines}

In conventional MMICs, the transmission lines, which have a characteristic impedance of $50 \Omega$, are widely used, and low- 


$$
\mathrm{Z}_{\mathbf{0}}=\mathbf{5 0} \Omega
$$

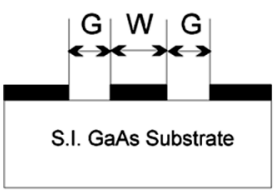

a 1)

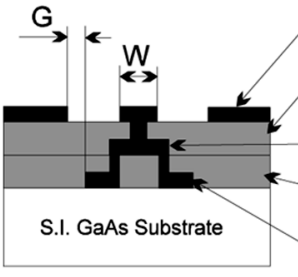

a 2)

.
$\mathrm{Z}_{0}>\mathbf{5 0} \Omega$

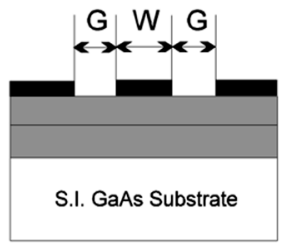

b 1)

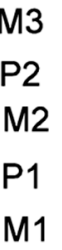

M1

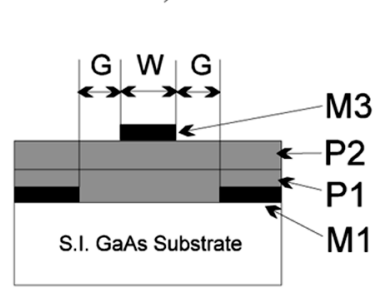

b 2)

$$
\mathbf{Z}_{0}<50 \Omega
$$

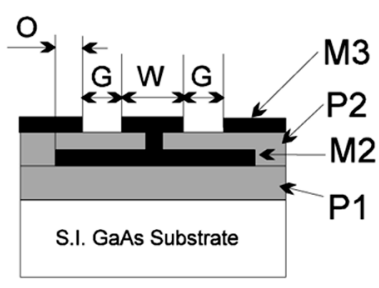

c 1)

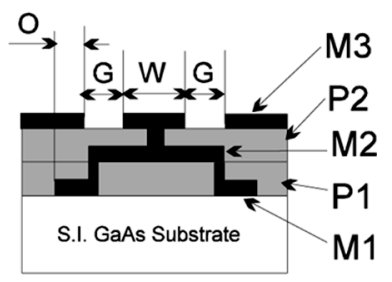

c 2)

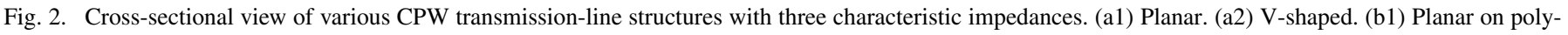
imide. (b2) Elevation. (c1) Overlap with 2 metal layers. (c2) V-shaped with overlap.

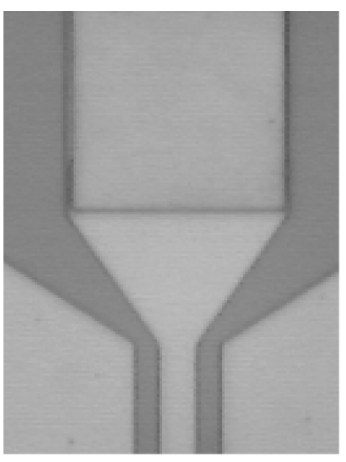

a)

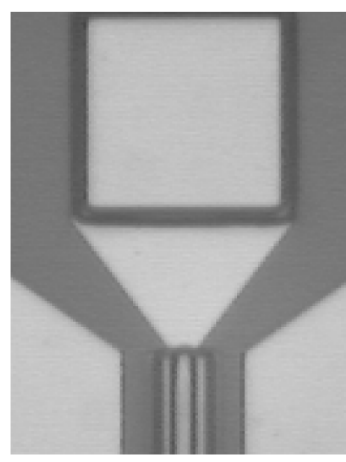

b)
Fig. 3. Micrographs of fabricated: (a) planar and (b) V-shaped CPW transmission lines.

loss compact transmission lines are desirable. However, conventional planar CPW transmission lines are lossy due to high signal loss at the edge of the conductor, which is caused by current crowding effects. Here, we demonstrate that by utilizing multilayer technology, low-loss V-shaped CPW transmission lines can be easily constructed. The cross-sectional view of a conventional and V-shaped CPW transmission line are shown in Fig. 2(a1) and (a2).

Three CPW transmission lines were designed to have a $Z_{0}$ of $50 \Omega$. The first one $(W=20 \mu \mathrm{m}, G=15 \mu \mathrm{m})$ is a conventional planar CPW transmission line on GaAs, while the two others are multilayer $\mathrm{V}$-shaped CPWs with the width of the top center line as $W=8$ and $12 \mu \mathrm{m}$ [see Figs. 2(a1) and Fig. 2(a2)]. All the transmission lines are 2-mm long. Fig. 3 shows micrographs of fabricated conventional and $\mathrm{V}$-shaped CPW transmission lines.

The effective dielectric constant $\left(\varepsilon_{\text {reffective }}\right)$ of the V-shaped transmission line are less than that of the planar one, as shown in Fig. 4(a). This is because the V-shaped design allows more electric flux lines in the low-permittivity polyimide layer and the air, which also even reduces the dissipation loss. A comparison of dissipation loss between the fabricated conventional

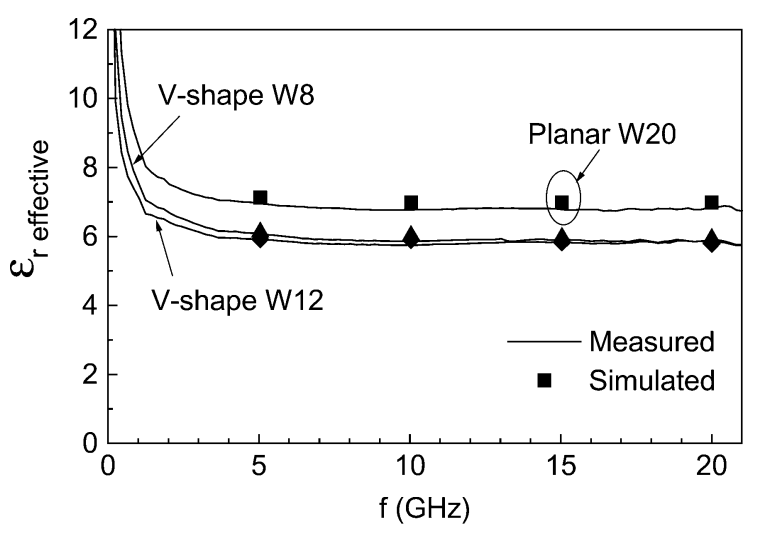

a)

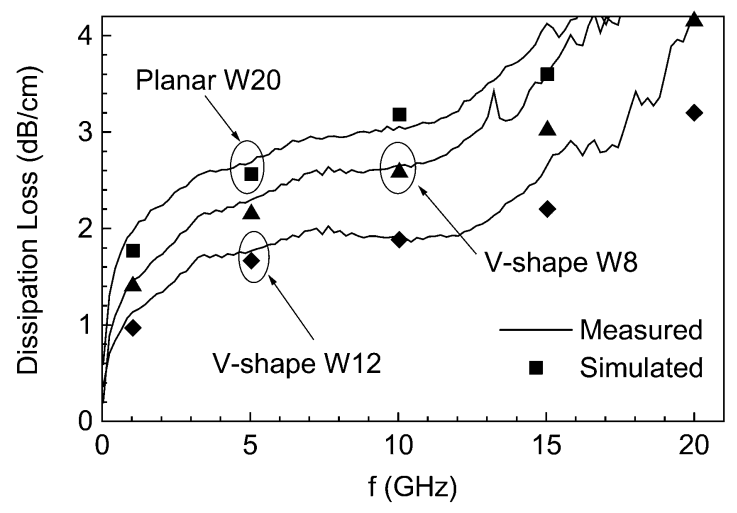

b)

Fig. 4. Simulated and measured results for: (a) effective dielectric constant $\varepsilon_{\text {reffective }}$ and (b) dissipation loss of a planar $(W=20 \mu \mathrm{m}, G=15 \mu \mathrm{m}$ ) and two V-shaped (W12: $W=12 \mu \mathrm{m}, G=15 \mu \mathrm{m}$ and W8: $W=8 \mu \mathrm{m}$, $G=10 \mu \mathrm{m}) \mathrm{CPW}$ transmission lines with $Z_{0}=50 \Omega$.

and V-shaped CPW transmission lines is shown in Fig. 4(b). It can be seen that both of the V-shaped CPW transmission lines are less lossy than the conventional planar version, in spite of having a thinner center conductor width. This was achieved by 


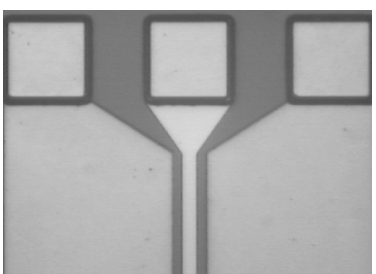

Fig. 5. Micrograph of a fabricated CPW transmission line with the center conductor elevated by $5-\mu \mathrm{m}$ dielectric layer, which is formed using metal 1 and metal 3 layers [see Fig. 2(b2)].

a proper design of the $\mathrm{V}$-shaped transmission lines, which effectively disperse the current within the conductors, thus eliminating the current crowding effect.

\section{B. High-Impedance $\left(Z_{0}>50 \Omega\right)$ Transmission Lines}

The characteristic impedance of CPW transmission lines can be increased simply by lifting (elevating) entire CPW lines with a low-permittivity polyimide layer, as shown in Fig. 2(b1). Elevation of the center line is also another simple design to increase $Z_{0}$ due to the reduction of its capacitance to the ground (see Fig. 2(b2) and Fig. 5). Here, we demonstrate how the described technique is effective. The dimension of the transmission lines shown in Fig. 2(b1) and (b2) were chosen as that of the conventional $50-\Omega \mathrm{CPW}$ transmission lines formed on the GaAs substrate $(W=20 \mu \mathrm{m}$ and $G=15 \mu \mathrm{m})$. The transmission lines are 2 -mm long.

The results in Fig. 6 show that the characteristic impedance increases from 50 to $65 \Omega$, i.e., by $30 \%$. Even higher impedance can be realized easily by widening the gap between the center conductor and ground planes or by increasing the thickness of the polyimide layer. Comparing the planar structure on the GaAs substrate (Fig. 2(a1), $\varepsilon_{r}=7$ ) with the same structure on top of the polyimide layer (Fig. 2(b1), $\varepsilon_{r}=4$ ), most of the electric flux lines remain in a low-permittivity polyimide and the air resulting in low effective dielectric constant, as shown in Fig. 6(b). This also reduces the dissipation loss. At $10 \mathrm{GHz}$, the structure on the polyimide gives $0.5-\mathrm{dB} / \mathrm{cm}$ lower loss than that on GaAs [see Figs. 4(b) and 6(c)].

\section{Low-Impedance $\left(Z_{0}<50 \Omega\right)$ Transmission Lines}

For the MMICs, low-impedance transmission lines have been shown to be useful components, especially in matching networks. In conventional MMICs, the transmission lines typically have a characteristic impedance of $40-100 \Omega$, while the microwave device themselves have a much lower input impedance. This imposes a requirement on the transmission-line design since low-impedance lines cannot readily be used. To design a coplanar transmission line with extra low impedance requires a very narrow slot between the conductors. However, at high frequency, the signal loss at the edge of the conductor can be high due to the current crowding effects. Various methods to overcome this problem and to realize low-loss lines and small compact microstrip lines have been proposed. In this study, we demonstrate that by employing multilayer structures, a low-impedance low-loss transmission line can easily be constructed. This was achieved by proper design of the transmission-line structure in which the bottom part of

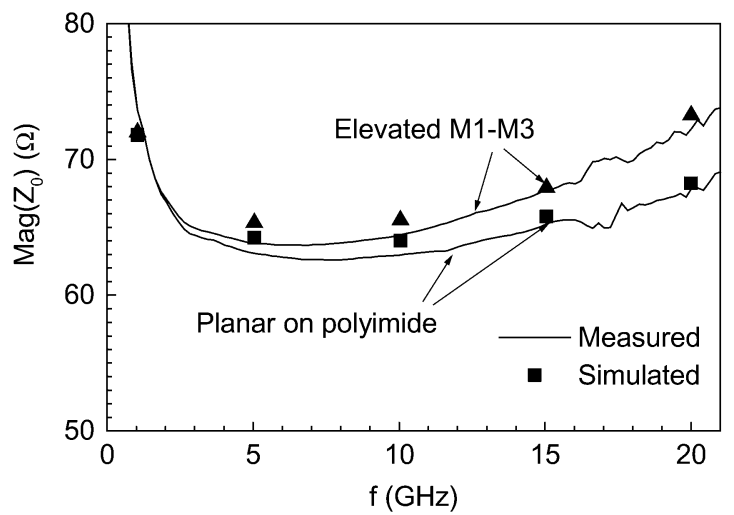

a)

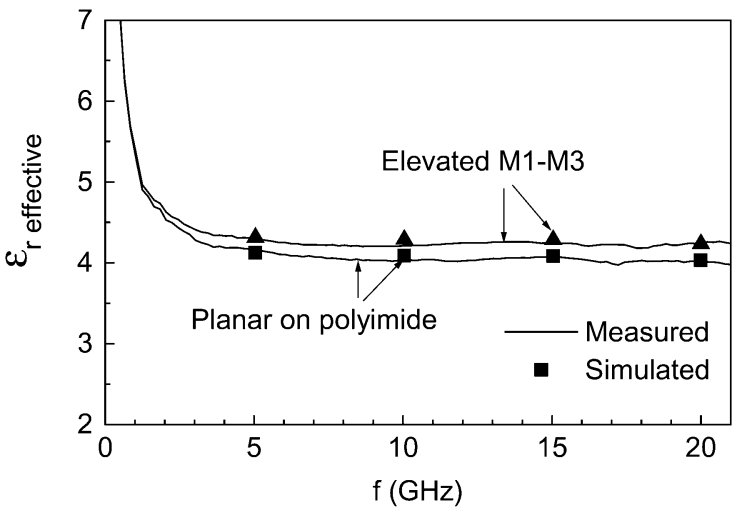

b)

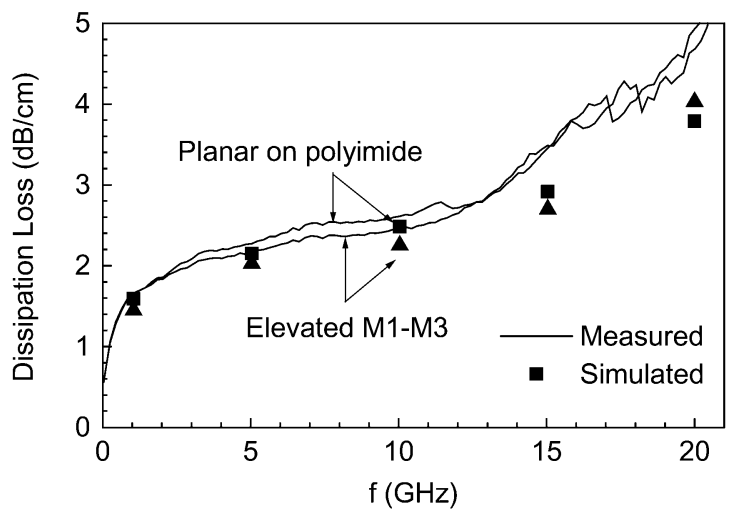

C)

Fig. 6. Simulated and measured results for: (a) characteristic impedance, (b) effective dielectric constant, and (c) dissipation loss of a planar CPW line on a polyimide and a CPW line with the center conductor elevated as shown in Fig. 2(b1) and 2(b2) ( $W=20 \mu \mathrm{m}$ and $G=15 \mu \mathrm{m}$ for both of the lines).

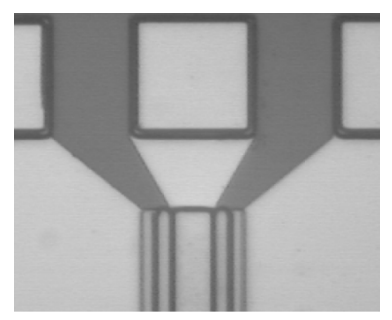

Fig. 7. Micrograph of a fabricated low-impedance V-shaped CPW transmission line.

the center line is overlapping the ground planes, increasing the capacitance to the ground, thus reducing the characteristic 


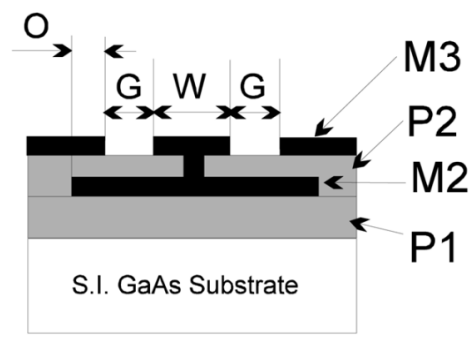

a)

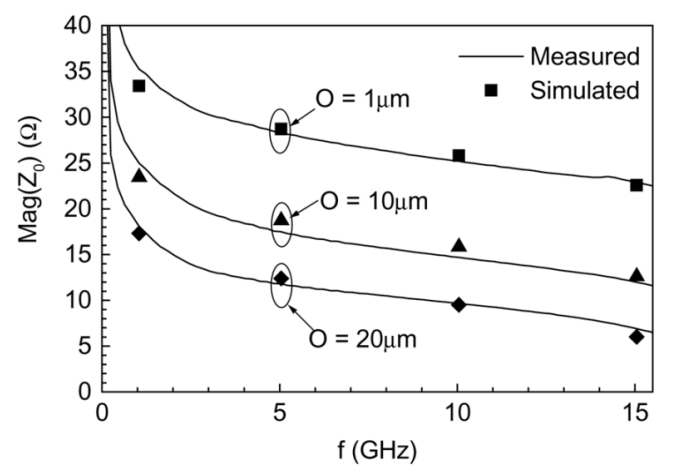

b)

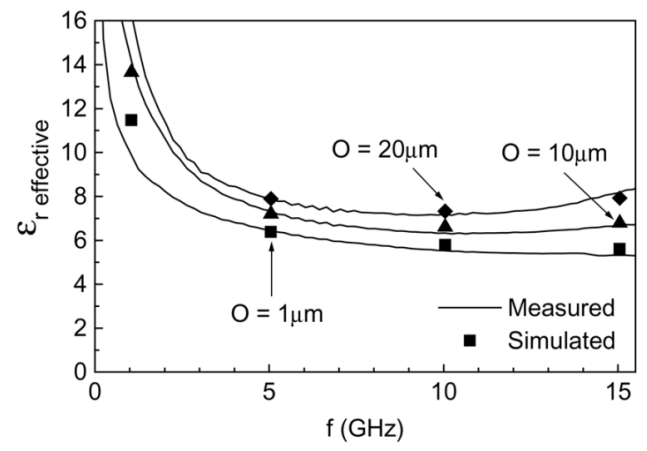

c)

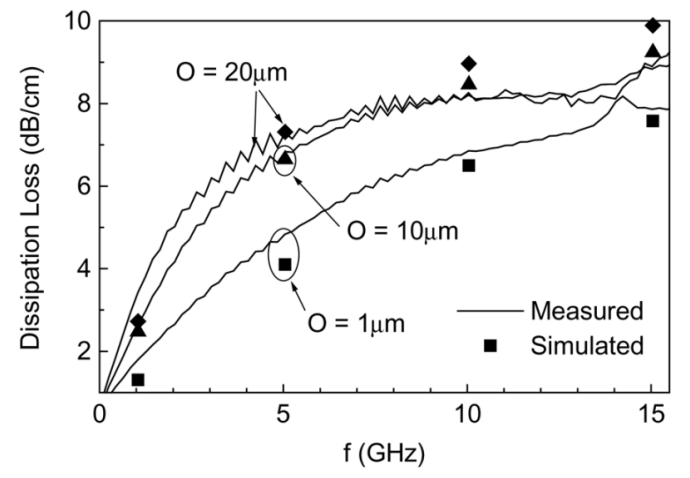

(d)

Fig. 8. (a) Cross-sectional view of a low-impedance CPW transmission line using two metal layers and simulated and measured results for its: (b) characteristic impedance, (c) effective dielectric constant, and (d) dissipation loss ( $W=40 \mu \mathrm{m}, G=15 \mu \mathrm{m}$ and the overlap size as 1,10 , and $20 \mu \mathrm{m}$ ).

impedance. V-shaped structures also allow the current to be effectively dispersed within the conductor, thus eliminating the current crowding effect. The structures that have been considered are shown in Fig. 2(c1) and (c2). Fig. 7 shows a fabricated low-impedance transmission line. All the transmission lines are 2-mm long.

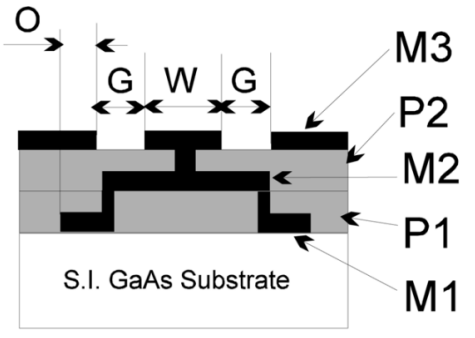

a)

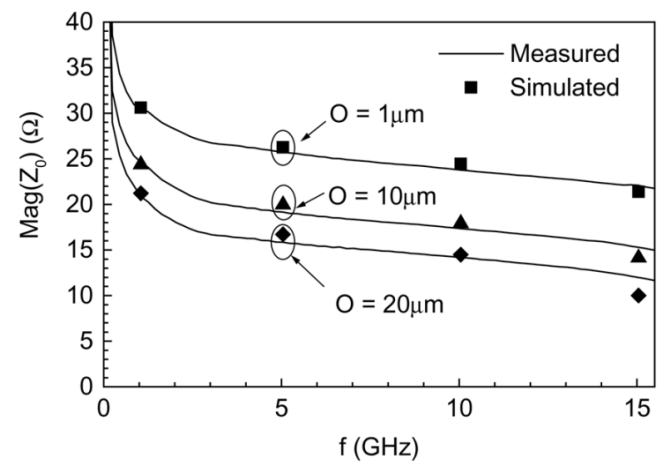

b)

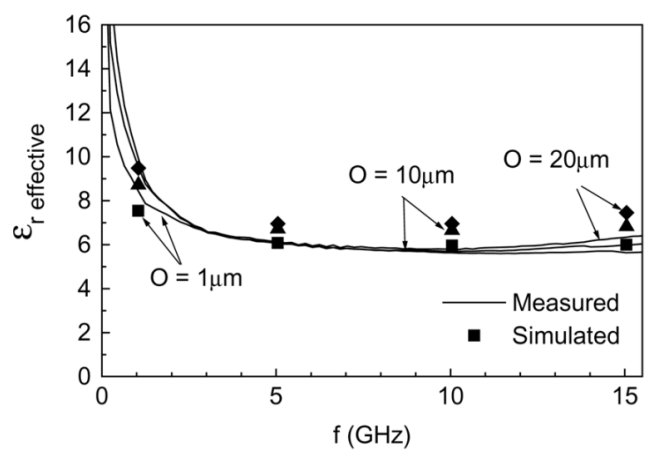

c)

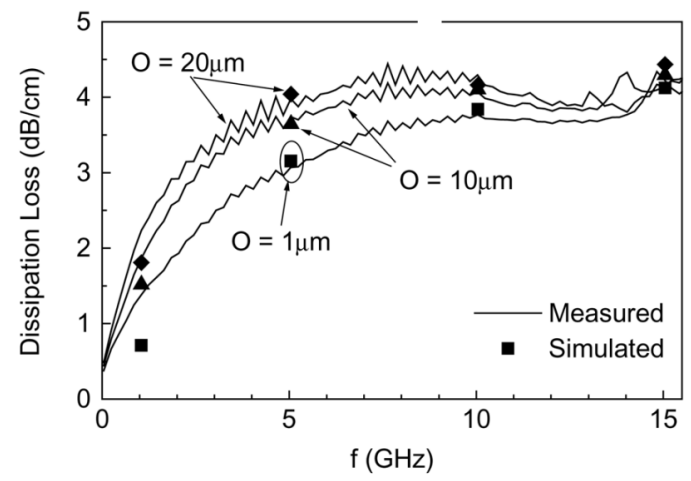

d)

Fig. 9. (a) Cross-sectional view of a low-impedance CPW transmission line using three metal layers and simulated and measured results for its: (b) characteristic impedance, (c) effective dielectric constant, and (d) dissipation loss ( $W=40 \mu \mathrm{m}, G=15 \mu \mathrm{m}$ and the overlap size as 1,10 , and $20 \mu \mathrm{m}$ ).

Fig. 8 shows the cross-sectional view of low-impedance transmission lines using two metal layers and their measured parameters, and Fig. 9 shows transmission lines realized by three metal layers. The results clearly show that with appropriate overlap design of transmission lines, various low impedance can be achieved for circuit matching. For example, a 


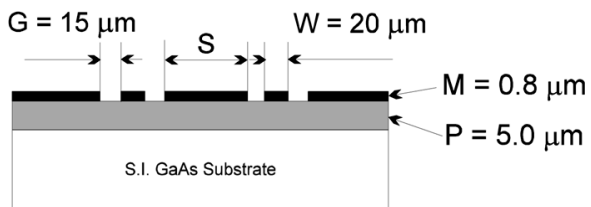

Fig. 10. Cross-sectional view of two adjoining transmission lines separated by the conductor having a width of $S(W=20 \mu \mathrm{m}, G=15 \mu \mathrm{m})$.

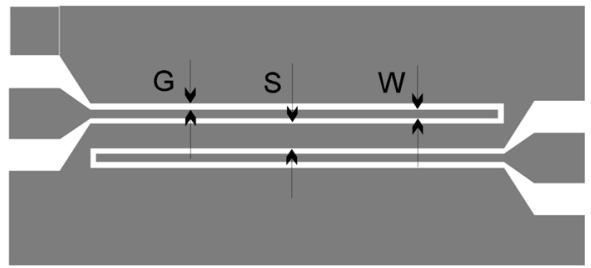

Fig. 11. Top view of two adjoining transmission lines separated by the conductor having a width of $S$ ( $W=20 \mu \mathrm{m}, G=15 \mu \mathrm{m})$.

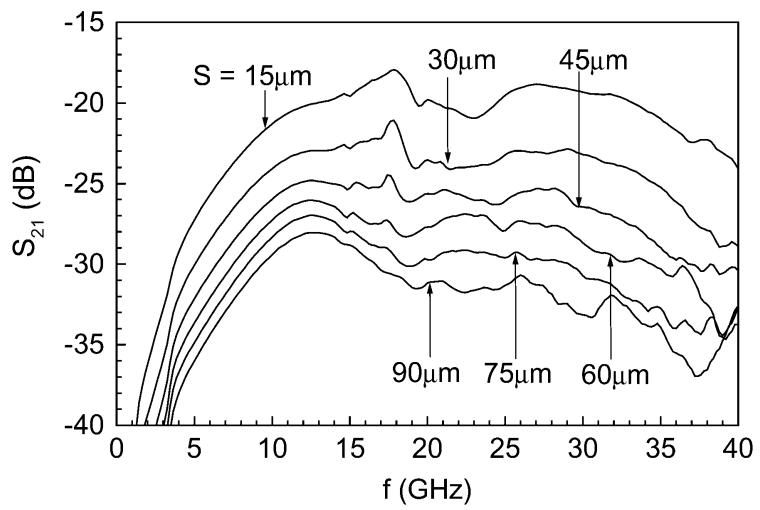

Fig. 12. Measured coupling between two adjoining transmission lines having various separation of $S$.

$20-\Omega$ impedance was achieved with a $1-\mu$ m overlap at $10 \mathrm{GHz}$. This can be even reduced to $10 \Omega$ by simply increasing the overlap to $20 \mu \mathrm{m}$, as shown in Fig. 8(b).

Comparing the data given in Figs. 8(d) and 9(d), it clearly demonstrates that, for the same impedance, the additional metal layer of the structure shown in Fig. 9(a) provides a 4-dB lower dissipation loss compared with the two metal-layer structure design given in Fig. 8(a). It is because the current is more effectively dispersed within the three metal layers, thus eliminating the current crowding effect. On the other hand, the electric field at the overlapping area is lower due to thicker polyimide resulting in lower dielectric loss.

\section{EFFECt of Unintentional HoRizontal COUPLing}

In order to investigate the effect of unintentional horizontal coupling, a set of two adjoining transmission lines was designed to investigate the effect of horizontal coupling on their parameters in order to identify an optimum separation where electromagnetic coupling can be neglected. Fig. 10 shows a crosssectional view of two adjoining transmission lines on top of a 5- $\mu$ m-thick polyimide layer stacked on the semi-insulating GaAs substrate. The transmission lines are conventional planar CPW transmission lines with the center conductor width $W=$ $20 \mu \mathrm{m}$ and the slot width $G=15 \mu \mathrm{m}$ and $1 \mathrm{~mm}$ long. The sep-

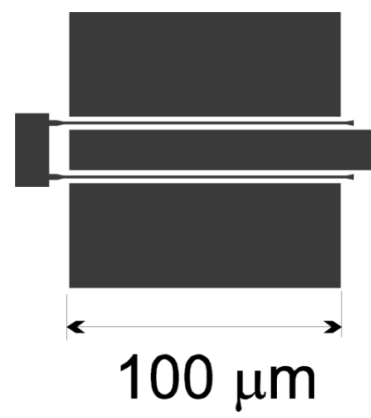

Fig. 13. Top view of metal contacts of a pHEMT used in the simulations for investigation of vertical coupling in multilayer MMICs (see Fig. 14).

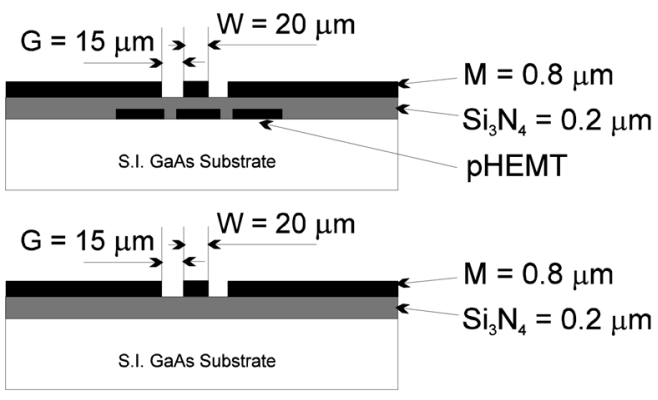

a)

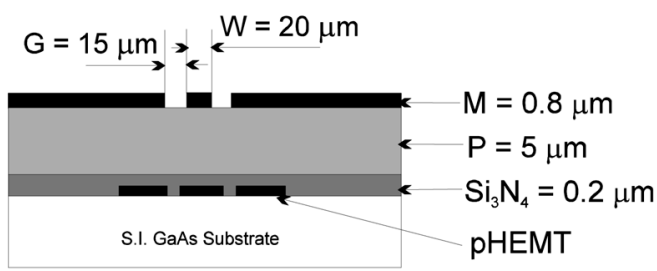

$G=15 \mu \mathrm{m}, c^{W}=20 \mu \mathrm{m}$

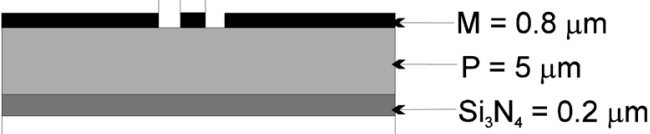

S.I. GaAs Substrate

b)

Fig. 14. Cross-sectional view of two sets of test structures for investigation of vertical coupling. Set (a) one with a pHEMT under the signal conductor and one without. Set (b), as in set (a), but with an addition of a 5- $\mu \mathrm{m}$-thick polyimide layer.

aration $(S)$ varies from $15 \mu \mathrm{m}(1 \times G)$ to $90 \mu \mathrm{m}(6 \times G)$ (see Fig. 11). In order to study this effect, six pairs of transmission lines with different spacing were designed, fabricated, and measured.

Fig. 12 shows measured isolation between the two adjoining transmission lines as a function of separation $(S)$. It can be clearly seen that the isolation can be improved by widening the distance between two transmission lines. However, the isolation is not improved with the spacing wider than $75 \mu \mathrm{m}(5 \times G)$, where $-30-\mathrm{dB}$ isolation is achieved. This isolation is sufficient for most applications.

\section{EFFect of Vertical Coupling}

Referring to the design of the 3-D CPW multilayer MMIC, it is necessary to investigate the vertical coupling between various 


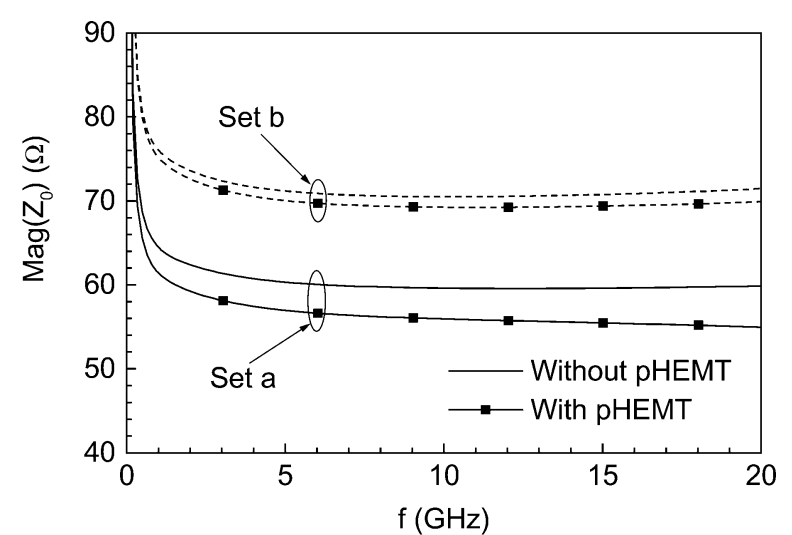

a)

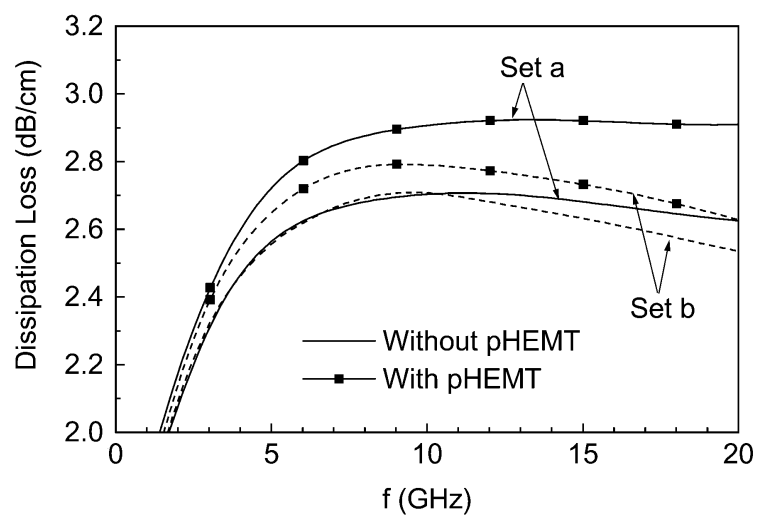

b)

Fig. 15. Simulated results of: (a) characteristic impedance and (b) dissipation loss of two set of transmission lines given in Fig. 14.

components. This is because of the possibility of over laying of transmission lines with other conductors, which can be metal contacts of active devices such as pHEMTs. In order to keep the MMIC chip area small, the separation between transmission lines and conductor elements of different layers should be as small as possible. However, this should be a problem if there is a strong unintentional vertical coupling between them, degrading the isolation between components within the MMIC chip.

The effect of this coupling on the characteristic impedance and dissipation loss of the transmission line that crosses a pHEMT have been investigated, as has how a 5 - $\mu$ m-thick polyimide layer can provide sufficient isolation in order to minimize the effect of vertical coupling. In this study, a CPW transmission line is formed on the top metal layer and metal contacts of a pHEMT are formed by the bottom metal layer directly under a CPW transmission line. Fig. 13 shows the top view of a pHEMT employed for this investigation. Two sets of test structures were designed to investigate the effect of vertical coupling, which are shown in Fig. 14. The test structures of set (a) is comprised of a semi-insulating GaAs substrate, pHEMT devices, silicon nitrite passivation layer, and a CPW transmission line on the top level, while set (b) has two extra polyimide layers with a total thickness of $5 \mu \mathrm{m}$ above an $\mathrm{Si}_{3} \mathrm{~N}_{4}$ layer. The CPW transmission line has a $20-\mu \mathrm{m}$-wide center line, $15-\mu \mathrm{m}$ gap to ground conductors, and is 1-mm long.

Simulations and characterization of the two sets of transmission lines were carried out with the help of the 2.5-D electromagnetic simulator Momentum. From the results shown in Fig. 15, it can be seen that set (a) shows approximately $10 \%$ decrease in characteristic impedance and $10 \%$ rise in the dissipation loss at $10 \mathrm{GHz}$. The reduction of the characteristic impedance is due to the increase of the capacitance of the line, which is caused by additional metal contacts of the pHEMT. The extra loss is due to the fact that some of the power is coupled to the metal contacts (pHEMT) located underneath the transmission line. However, if one covers the substrate with a 5 - $\mu \mathrm{m}$-thick polyimide layer, the impedance changes very slightly and the loss increases only by $3 \%$. This effect can be understood since the thick polyimide layer prevents electric flux penetration to conductors on the GaAs substrate. This thick polyimide also provides a good isolation of the structure.

These results clearly demonstrate that a 5- $\mu \mathrm{m}$-thick polyimide layer can provide sufficient isolation so that transmission lines of the top layer can cross the area above the pHEMT device without significant change of its parameters. Careful design of the integration is also necessary to ensure minimum signal loss.

\section{CONCLUSION}

Newly developed complete tools for low-loss 3-D CPW transmission lines on semi-insulating GaAs substrates have been designed, fabricated, and characterized. The transmission lines have been fabricated using three layers of metals and two layers of sandwich polyimide. The results have shown that a variety of low-loss CPW transmission lines for MMICs, which provide good performance to meet various circuit requirements, can easily be constructed by utilizing multilayer technique. This approach gives microwave engineers the flexibility in designing multilayer circuits with improved performance.

Furthermore, the unintentional coupling between CPW transmission lines both horizontally and vertically with other conductor elements embedded in the layout needs careful consideration for optimum design integration.

\section{ACKNOWLEDGMENT}

The authors would like to thank K. Williams, University of Manchester, Manchester, U.K., for his assistance in the RF measurements.

\section{REFERENCES}

[1] M. Riaziat, I. Zubeck, S. Bandy, and G. Zdasiuk, "Coplanar waveguides used in 2-18 GHz distributed amplifier," in IEEE MTT-S Int. Microw. Symp. Dig., Jun. 1986, pp. 337-338.

[2] R. W. Jackson, "Considerations in the use of coplanar waveguide for millimeter-wave integrated circuits," IEEE Trans. Microw. Theory Tech., vol. MTT-34, no. 12, pp. 1450-1456, Dec. 1986.

[3] T. Hirota, A. Minakawa, and M. Muraguchi, "Reduced size branchline and rat race hybrids for uniplanar MMIC's," IEEE Trans. Microw. Theory Tech., vol. 38, no. 3, pp. 270-275, Mar. 1990.

[4] K. C. Gupta, R. Grag, and R. Chada, Computer Aided Design of Microwave Circuits. Dedham, MA: Artech House, 1981, pp. 25-43.

[5] T. Shibata and E. Sano, "Characterization of MIS structure coplanar transmission lines for investigation of signal propagation in integrated circuits," IEEE Trans. Microw. Theory Tech., vol. 38, no. 7, pp. 881-890, Jul. 1990.

[6] W. R. Eisenstadt and Y. Eo, " $S$-parameter-based IC-interconnect transmission line characterization," IEEE Trans. Compon., Hybrid, Manuf. Technol., vol. 15, no. 4, pp. 483-490, Aug. 1992. 
[7] W. Durr, U. Erben, A. Schuppen, H. Dietrich, and H. Schumacher, "Investigation of microstrip and coplanar transmission lines on lossy silicon substrates without backside metallization," IEEE Trans. Microw. Theory Tech., vol. 46, no. 5, pp. 712-715, May 1998.

[8] W. Heinrich, J. Gerdes, F. J. Schmuckle, C. Rheifelder, and K. Strohm, "Coplanar passive elements on Si substrate for frequencies up to 110 GHz," IEEE Trans. Microw. Theory Tech., vol. 46, no. 5, pp. 709-712, May 1998.

[9] F. Schnieder, R. Doerner, and W. Heinrich, "High-impedance coplanar waveguides with low attenuation," IEEE Microw. Guided Wave Lett., vol. 6, no. 3, pp. 117-119, Mar. 1996.

[10] S.-H. Jeong, S.-J. Yoon, J.-G. Yook, S.-G. Lee, and Y.-J. Kim, "Elevated-CPW for high-speed digital interconnects," IEEE AP-S Int. Symp., pp. 541-544, 2001.

[11] H. Ogawa, T. Hasegawa, S. Banba, and H. Nakamoto, "MMIC transmission lines for multi-layered MMICs," in IEEE MTT-S Int. Microwave Symp. Dig., 1991, pp. 1067-1069.

[12] D. Budimir, Q. H. Wang, A. A. Rezazadeh, and I. D. Robertson, "V-shape CPW transmission lines for multilayer MMICs," Electron. Lett., vol. 31, pp. 1928-1929, Oct. 1995.

[13] C. Warns, W. Menzel, and H. Schumacher, "Transmission lines and passive elements for multilayer coplanar circuits on silicon," IEEE Trans. Microw. Theory Tech., vol. 46, no. 5, pp. 616-622, May 1998.

[14] J.-P. Raskin, G. Gauthier, L. P. Katehi, and G. M. Rebeiz, " $W$-band single-layer vertical transitions," IEEE Trans. Microw. Theory Tech., vol. 48, no. 1, pp. 161-164, Jan. 2000.

[15] Z. Lei and W. Menzel, "Broad-band microstrip-to-CPW transition via frequency-dependent electromagnetic coupling," IEEE Trans. Microw. Theory Tech., vol. 52, no. 5, pp. 1517-1522, May 2004.

[16] J. Papapolymerou, G. E. Ponchak, E. Dalton, A. Bacon, and M. M. Tentzeris, "Crosstalk between finite ground coplanar waveguides over polyimide layers for 3-D MMIC on Si substrates," IEEE Trans. Microw. Theory Tech., vol. 52, no. 4, pp. 1292-1301, Apr. 2004.

[17] V. T. Vo, L. Krishnamurthy, Q. Sun, A. A. Rezazadeh, and R. Sloan, "3-D low-loss coplanar waveguide transmission line structures with wide range of characteristic impedance for MMICs," in Proc. 35th Eur. Microw. Conf., Paris, France, Oct. 2005, pp. 4-7.

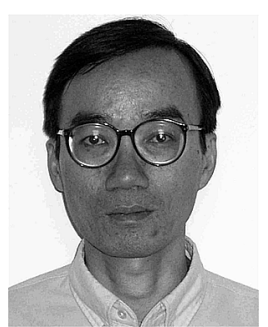

Van Tuyen Vo was born in Go Cong, Vietnam. $\mathrm{He}$ received the Diploma degree in electrical and electronic engineering from the Technical University of Budapest, Budapest, Hungary, in 1988, and the Ph.D. degree from the Hungarian Academy of Sciences, Budapest, Hungary, in 1995.

In 1988, he joined the Research Institute for Technical Physics, Hungarian Academy of Sciences. He had been involved in research and development on microwave GaAs devices and applications. In 2001, he joined the Department of Electronic Engineering, King's College, London, U.K., where he was engaged in research and development of zero-bias $\mathrm{Si}$ and GaAs planar doped barrier diodes for millimeterwave/microwave detectors/sensors. In 2003, he joined the School of Electrical and Electronic Engineering, University of Manchester, Manchester, U.K. He recent activities focus on 3-D MMIC design and technology.

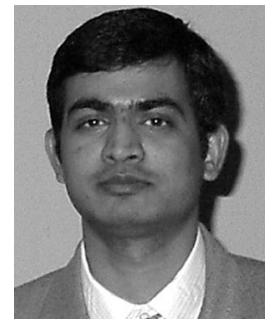

Lokesh Krishnamurthy ( $\mathrm{S}^{\prime} 01$ ) was born in Bangalore, India. He received the Diploma and B.Eng. degree in electronics and communication from Bangalore University, Bangalore, India, the M.Sc. degree in system-level integration from the University of Edinburgh, Edinburgh, U.K., and is currently working toward $\mathrm{Ph} . \mathrm{D}$. degree at the University of Manchester, Manchester, U.K.

$\mathrm{He}$ is currently with the Electromagnetics Centre, University of Manchester. His field of research is comprised of design, characterization, optimization, and integration of multilayer passive components for advanced MMIC technologies. He is also currently involved in the design and development of multilayer low-noise amplifiers based on pHEMTs. His other research interests include developing new methodologies for parameter extraction and characterization of both active and multilayer passive components.

Mr. Krishnamurthy was a recipient of the Overseas Research Scholarship in the U.K. and the Graduate Research Award presented at the European Microwave Week 2005, Paris, France.

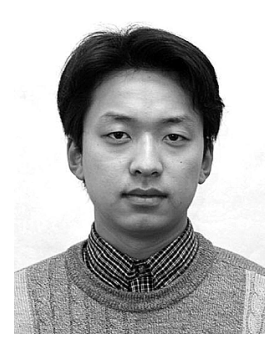

Qing Sun (S'04) was born in Taiyuan, Shanxi, China, in 1981. He received the B.S. degree in electronic engineering from Taiyuan University of Technology, Taiyuan, Shanxi, China, in 2002, the M.Sc. degree in communication engineering from the University of Manchester Institute of Science and Technology (UMIST), Manchester, U.K., in 2004, and is currently working toward the Ph.D. degree in microwave engineering at the University of Manchester, Manchester, U.K.

$\mathrm{He}$ is currently with the Electromagnetics Centre, University of Manchester. His research topic is novel MMIC based on multilayer CPW technology.

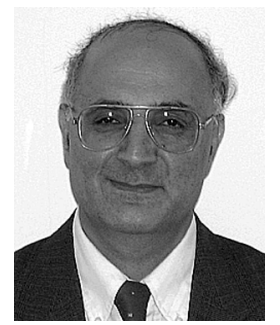

Ali A. Rezazadeh (M'90) is currently a Professor of microwave engineering with the School of Electrical and Electronic Engineering, University of Manchester, Manchester, U.K. He is also the Director of the Electromagnetics Research Centre, University of Manchester. From 1990 to July 2002, he was a Reader of microwaves and photonics and the Head of the Microwave Circuits and Devices Research Group, Department of Electronic Engineering, King's College, University of London, London, U.K. From 1983 to 1990, he was a Research Scientist with the GEC-Marconi Hirst Research Centre and became the Group Leader responsible for research and development into advanced heterojunction devices and circuits for high-speed and digital applications. He has taught many courses on engineering physics, microwave and photonics devices and circuits technology and components for MMICs and a short course on fundamentals of microwave power-amplifier designs annually held at the European Microwave Week Conference. He has authored several book chapters and conference proceedings and has authored or coauthored over 180 refereed journal and conference papers. His current research interests are in the area of design and technology of III-V circuits and devices including multilayer circuits for 3-D MMIC applications.

Prof. Rezazadeh is the chairman of the IEEE UKRI [Microwave Theory and Techniques (MTT)/Electron Device (ED)/Antennas and Propagation (AP)/Lasers and Electro-Optics (LEO)] joint Chapter. In 1993 he founded the IEEE International Symposium on High Performance Electron Devices for Microwave and Optoelectronic applications (EDMO). He was the recipient of numerous awards an in 2000, received the IEEE Third Millennium Medal. 